\title{
CANONICAL BUNDLES OF COMPLEX NILMANIFOLDS, WITH APPLICATIONS TO HYPERCOMPLEX GEOMETRY
}

\author{
María L. Barberis, Isabel G. Dotti and Misha Verbitsky ${ }^{1}$
}

\begin{abstract}
A nilmanifold is a quotient of a nilpotent group $G$ by a co-compact discrete subgroup. A complex nilmanifold is one which is equipped with a $G$-invariant complex structure. We prove that a complex nilmanifold has trivial canonical bundle. This is used to study hypercomplex nilmanifolds (nilmanifolds with a triple of $G$-invariant complex structures which satisfy quaternionic relations). We prove that a hypercomplex nilmanifold admits an HKT (hyperkähler with torsion) metric if and only if the underlying hypercomplex structure is abelian. Moreover, any $G$-invariant HKT-metric on a nilmanifold is balanced with respect to all associated complex structures.
\end{abstract}

\section{Introduction}

1.1. Canonical bundle of complex nilmanifolds. Let $G$ be a connected, simply connected nilpotent Lie group, and $\Gamma \subset G$ a discrete, co-compact subgroup. The quotient manifold $\Gamma \backslash G$ is called a nilmanifold. Clearly, $G$ acts on $\Gamma \backslash G$ transitively (from the right). Nilmanifolds are often defined as compact manifolds with a transitive action of a nilpotent Lie group (see e.g. $[\mathrm{H}]$ ). In this case, the above definition becomes a theorem, proven by Mal'cev, who introduced this notion in 1949, in the influental paper $[\mathrm{M}]$.

If $N=\Gamma \backslash G$ is equipped with a complex structure $\mathcal{I}$ induced by a left-invariant complex structure on $G,(N, \mathcal{I})$ is called a complex nilmanifold.

It is important to note that $G$ is not necessarily a complex Lie group. Indeed, for $G$ to be a complex Lie group, both left and right translations on $G$ must be holomorphic. In many examples of complex nilmanifolds, even the simplest ones (such as a Kodaira surface), this condition is not satisfied.

Geometry of nilmanifolds is an important subject, much studied since Mal'cev and Mostow started this work. The complex nilmanifolds are much less understood. There are many papers dealing with special cases of nilpotent Lie groups and particular discrete co-compact subgroups, where the complex invariants of the corresponding nilmanifolds (deformation space, Frölicher spectral sequence, and so on) have been computed. However, general results about complex nilmanifolds remain scarce. Indeed, nilmanifolds serve mostly as a rich source of counterexamples to all kinds of general conjectures in geometry and topology ([Ab], [CFG1], [CFL]).

In the present paper, we prove that the canonical bundle of any complex nilmanifold is holomorphically trivial (Theorem 2.7). This condition is quite strong. For instance, any compact complex surface with trivial canonical bundle is isomorphic to a K3

Received by the editors February 14, 2008.

${ }^{1}$ Misha Verbitsky is partially supported by a CRDF grant RM1-2354-MO02. 
surface, a torus, or a Kodaira surface; the first two are Kähler, and the latter is a nilmanifold.

Kähler manifolds with trivial canonical bundle play an important role in mathematics and physics, due to the nice behavior of their deformation spaces, guaranteed by Bogomolov-Tian-Todorov theorem. This theorem states that the deformations of a compact Kähler manifold with trivial canonical bundle are non-obstructed, and the deformation space is smooth.

For nilmanifolds obtained as quotients of complex nilpotent groups ("complex parallelisable nilmanifolds"), this is known to be false, as S. Rollenske proved ([Ro2]). For other classes of nilmanifolds, for instance hypercomplex nilmanifolds, some version of Bogomolov-Tian-Todorov theorem could still be true. In fact, the key ingredient of the proof of Bogomolov-Tian-Todorov theorem, the so-called Tian-Todorov lemma $([\mathrm{BK}])$, remains valid for any complex manifold with trivial canonical bundle. The rest of the proof, sadly, does not work, because it requires the degeneration of a Frölicher spectral sequence, and (as shown in [CFG2] and [Ro1]), this sequence can be arbitrarily non-degenerate. Still, the vast empirical evidence (see e.g. [MPPS], $[\mathrm{Po}]$ ) shows that some analogue of Tian-Todorov formalism could exist on some (or all) nilmanifolds.

For general compact non-Kähler manifolds with trivial canonical bundle, an analogue of Bogomolov-Tian-Todorov theorem is known to be false. In $[\mathrm{G}]$, it was shown that the deformation space of a locally homogeneous manifold $S L(2, \mathbb{C}) / \Gamma$ can be obstructed, for a cocompact and discrete subgroup $\Gamma \subset S L(2, \mathbb{C})$.

One of the first examples of a complex manifold with obstructed deformations was constructed by A. Douady, in [Do]. Douady used an Iwasawa manifold which is a quotient $M:=G / \Gamma$, with $G$ the group of complex upper triangular $3 \times 3$-matrices, and $\Gamma$ the group of upper triangular matrices with coefficients in Gaussian integers.

Douady proved that a product $M \times \mathbb{C} P^{1}$ has obstructed deformation space. In [Re], Douady's construction was generalized, using Massey operations on cohomology of $M$.

Another proof of triviality of the canonical bundle of a nilmanifold is given in [CG].

1.2. Hypercomplex nilmanifolds. An almost hypercomplex manifold is a smooth manifold $M$ equipped with three operators $\mathcal{I}, \mathcal{J}, \mathcal{K} \in \operatorname{End}(T M)$ satisfying the quaternionic relations $\mathcal{I} \circ \mathcal{J}=-\mathcal{J} \circ \mathcal{I}=\mathcal{K}, \mathcal{I}^{2}=\mathcal{J}^{2}=\mathcal{K}^{2}=-\operatorname{Id}_{T M}$. The operators $\mathcal{I}$, $\mathcal{J}, \mathcal{K}$ define almost complex structures on $M$; if these almost complex structures are integrable, $M$ is called hypercomplex. A hypercomplex manifold is equipped with a whole 2-dimensional sphere of complex structures.

Hypercomplex manifolds were defined by C.P. Boyer ([Bo]), who gave a classification of compact hypercomplex manifolds for $\operatorname{dim}_{\mathbb{H}} M=1$. Many interesting examples of hypercomplex manifolds were found in the 90-ies, see e.g. [J], [PP], [BD1]. Independently (and earlier) some of these constructions were obtained by string physicists; see e.g. [SSTV].

As Obata has shown ([Ob]), a hypercomplex manifold admits a (necessarily unique) torsion-free connection, preserving $\mathcal{I}, \mathcal{J}, \mathcal{K}$. The converse is also true: if an almost hypercomplex manifold admits a torsion-free connection preserving the quaternionic action, it is hypercomplex. This implies that a hypercomplex structure on a manifold can be defined as a torsion-free connection with holonomy in $G L(n, \mathbb{H})$. 
Connections with restricted holonomy is one of the central notions in Riemannian geometry, due to Berger's classification of irreducible holonomy of Riemannian manifolds. However, a similar classification exists for a general torsion-free connection ([MS]). In the Merkulov-Schwachhöfer list, only three subroups of $G L(n, \mathbb{H})$ occur. In addition to the compact group $S p(n)$ (which defines hyperkähler geometry), also $G L(n, \mathbb{H})$ and its commutator $S L(n, \mathbb{H})$ appear, corresponding to hypercomplex manifolds and hypercomplex manifolds with trivial determinant bundle, respectively. Both of these geometries are interesting, rich in structure and examples, and deserve detailed study.

Not much is known about $S L(n, \mathbb{H})$-manifolds. It is easy to see that $(M, \mathcal{I})$ has holomorphically trivial canonical bundle, when $(M, \mathcal{I}, \mathcal{J}, \mathcal{K})$ is a hypercomplex manifold with holonomy in $S L(n, \mathbb{H})$ ([V2]). For a hypercomplex $S L(n, \mathbb{H})$-manifold admitting a special kind of quaternionic Hermitian metric called HKT metric, a version of Hodge theory was constructed ([V1]). Using this result, it was shown that a compact hypercomplex manifold with trivial canonical bundle has holonomy in $S L(n, \mathbb{H})$, if it admits an HKT-structure ([V2])

It is not clear whether the last condition is necessary: for all known examples of hypercomplex manifolds with trivial canonical bundle, holonomy lies in $S L(n, \mathbb{H})$.

In the present paper, we prove that holonomy $\operatorname{Hol}(\nabla)$ of a hypercomplex nilmanifold always lies in $S L(n, \mathbb{H}$ ) (Theorem 3.2)

As shown in $[\mathrm{AM}]$, locally $\operatorname{Hol}(\nabla) \subset S L(n, \mathbb{H})$ is equivalent to vanishing of the Ricci curvature of $\nabla$. However, the vanishing of Ricci curvature is weaker than $\operatorname{Hol}(\nabla) \subset$ $S L(n, \mathbb{H})$. Consider for example the Hopf manifold $H=\mathbb{Z} \backslash\left(\mathbb{H}^{n}-0\right)$. The Obata connection on $H$ is obviously flat, hence the Ricci curvature vanishes. However, $\operatorname{Hol}(\nabla)$ does not lie in $S L(n, \mathbb{H})$. In fact, the canonical bundle of $H$ is holomorphically non-trivial, and has no non-zero sections (see Subsection 2.2).

We give an independent proof of vanishing of Ricci curvature of a hypercomplex nilmanifold (Section 3).

1.3. Abelian complex structures. A complex nilmanifold $(N, \mathcal{I})$, with $N=\Gamma \backslash G$, gives rise to a splitting

$$
\mathfrak{g} \otimes_{\mathbb{R}} \mathbb{C}=\mathfrak{g}^{0,1} \oplus \mathfrak{g}^{1,0},
$$

where $\mathfrak{g}^{0,1}, \mathfrak{g}^{1,0}$ are the eigenspaces of the induced complex structure on the Lie algebra $\mathfrak{g}$ of $G$. By Newlander-Nirenberg theorem, the almost complex structure $\mathcal{I}$ is integrable if and only if $\mathfrak{g}^{1,0}$ is a complex subalgebra of $\mathfrak{g} \otimes_{\mathbb{R}} \mathbb{C}$. $(N, \mathcal{I})$ is called abelian if the Lie subalgebra $\mathfrak{g}^{1,0}$ is abelian.

Abelian complex structures were introduced in [B1], and much studied since then (see, for example, [BDM], [BD1]). There are strong restrictions to the existence of such structures. In fact, it has been shown by $[\mathrm{Pe}]$ that the Lie algebra must be two-step solvable. However, a complete classification is still unknown, though there exist some partial results ([BD2]). The complex geometry of nilmanifolds with abelian complex structures is much more accessible than the general case. In particular, the Dolbeault cohomology of an abelian nilmanifold can be expressed in terms of the corresponding Lie algebra cohomology $([\mathrm{CF}],[\mathrm{CFGU}])$, and the same is true for the deformation space ([MPPS], [V2], [CFP]).

This notion is specially convenient when applied to hypercomplex nilmanifolds. If $(N, \mathcal{I}, \mathcal{J}, \mathcal{K})$ is a hypercomplex nilmanifold, abelianness of the complex structure 
$\mathcal{I}$ is equivalent to the abelianness of $\mathcal{J}$ and $\mathcal{K}$ ([DF3]). Some results on abelian hypercomplex structures can be found in [DF1], [B3].

1.4. HKT-structures on nilmanifolds. Let $(M, \mathcal{I}, \mathcal{J}, \mathcal{K})$ be a hypercomplex manifold. A "hyperkähler with torsion" (HKT) metric on $M$ is a special kind of a quaternionic Hermitian metric, which became increasingly important in mathematics and physics during the last seven years. HKT-metrics were introduced by P. S. Howe and G. Papadopoulos ([HP]) and much discussed in the physics and mathematics literature since then. See $[\mathrm{GP}]$ for a treatment of HKT-metrics written from a mathematical point of view. The term "hyperkähler metric with torsion" is actually misleading, because an HKT-metric is not hyperkähler. This is why we prefer to use the abbreviation "HKT-manifold".

A quaternionic Hermitian metric is a Riemannian metric which is Hermitian under $\mathcal{I}, \mathcal{J}$ and $\mathcal{K}$. There are three Hermitian forms associated with such a metric $g$ :

$$
\omega_{\mathcal{I}}=g(\cdot, \mathcal{I} \cdot), \quad \omega_{\mathcal{J}}=g(\cdot, \mathcal{J} \cdot), \quad \omega_{\mathcal{K}}=g(\cdot, \mathcal{K} \cdot) .
$$

When these forms are closed $(M, \mathcal{I}, \mathcal{J}, \mathcal{K}, g)$ is called a hyperkähler manifold. In this case, $M$ is also holomorphically symplectic; indeed, the form $\omega_{\mathcal{J}}+\sqrt{-1} \omega_{\mathcal{K}}$ lies in $\Lambda^{2,0}(M, \mathcal{I})$. Being closed, this $(2,0)$ form is necessarily holomorphic.

The converse is also true: by Calabi-Yau theorem ([Bes], [Yau]), a compact holomorphically symplectic Kähler manifold admits a hyperkähler metric, which is unique in a given Kähler class. In algebraic geometry, the word "hyperkähler" is often used as a synonym to "holomorphically symplectic".

The condition $d\left(\omega_{\mathcal{J}}+\sqrt{-1} \omega_{\mathcal{K}}\right)=0$ is equivalent to hyperkählerianness. A weaker condition

$$
\partial\left(\omega_{\mathcal{J}}+\sqrt{-1} \omega_{\mathcal{K}}\right)=0
$$

is often more useful. A quaternionic Hermitian metric $g$ which satisfies (1.1) is called HKT (hyperkähler with torsion). As in the Kähler case, an HKT metric locally has a potential (see $[\mathrm{BS}]$ ).

For abelian hypercomplex nilmanifolds, any left-invariant quaternionic Hermitian metric is automatically HKT ([DF2]) and for 2-step nilmanifolds a converse result was proven in [DF2]. Using the triviality of the canonical bundle and the hypercomplex version of Hodge theory [V1], we generalize the previous result, showing that any hypercomplex nilmanifold which admits a left-invariant HKT-metric is in fact abelian (Theorem 4.6). In $[\mathrm{GP}]$ the question whether any compact hypercomplex manifold admits an HKT metric was posed. In particular, a negative answer to this question is given by a non-abelian hypercomplex nilmanifold, since it has been shown in [FG] that existence of any HKT-metric compatible with a left-invariant hypercomplex structure implies existence of a left-invariant one. In $\S 4.3$ a family of non-abelian hypercomplex nilmanifolds is exhibited (see also the nilmanifold considered in the Remark of $\S 4$ in [DF1] and Lemma 3.1 in [FG]).

We also obtain, as a consequence of Theorem 4.6 and Proposition 4.11, that any invariant HKT-metric on a hypercomplex nilmanifold is balanced with respect to all underlying complex structures. 


\section{Geometry of complex nilmanifolds}

\subsection{Complex nilmanifolds: basic properties.}

Definition 2.1: A nilmanifold is a quotient $\Gamma \backslash G$ of a connected simply connected nilpotent Lie group $G$ by a co-compact discrete subgroup $\Gamma$.

By Mal'čev theorem ([M]), for any simply connected nilpotent Lie group $G$ with rational structure constants there is a lattice $\Gamma$ of maximal rank.

Let $G$ be a real Lie group, equipped with a left-invariant almost complex structure $\mathcal{I}$, acting on its Lie algebra as $I: \mathfrak{g} \longrightarrow \mathfrak{g}, I^{2}=-$ Id. It is well known that $\mathcal{I}$ is integrable if and only if the $\sqrt{-1}$-eigenspace $\mathfrak{g}^{0,1} \subset \mathfrak{g}_{\mathbb{C}}:=\mathfrak{g} \otimes_{\mathbb{R}} \mathbb{C}$ is a subalgebra of $\mathfrak{g}_{\mathbb{C}}$. In this situation, we shall say that $G$ is equipped with a left-invariant complex structure. When $I: \mathfrak{g} \longrightarrow \mathfrak{g}$ satisfies the condition $[I x, I y]=[x, y]$ for any $x, y \in \mathfrak{g}, \mathcal{I}$ is integrable and it is called an abelian complex structure. In this case, it turns out that $\mathfrak{g}^{1,0} \subset \mathfrak{g} \otimes_{\mathbb{R}} \mathbb{C}$ is a complex abelian subalgebra of $\mathfrak{g} \otimes_{\mathbb{R}} \mathbb{C}$.

Let $G$ be a nilpotent Lie group with a left-invariant complex structure $\mathcal{I}$. According to Theorem 1.3 in [S], there exist left-invariant $(1,0)$-forms $\omega_{1}, \ldots, \omega_{n}$ and smooth 1 -forms $\eta_{1}^{i}, \ldots, \eta_{i-1}^{i}$ on $G$ for $2 \leqslant i \leqslant n$, such that

$$
d \omega_{i}=\sum_{j<i} \eta_{j}^{i} \wedge \omega_{j} .
$$

We prove next an algebraic lemma, which will be useful to prove that a hypercomplex nilmanifold is Ricci flat (see Corollary 3.4). Its proof makes use of the existence of the above basis of $(1,0)$-forms.

Lemma 2.2: Let $\mathcal{J}$ be a complex structure on a nilpotent Lie algebra $\mathfrak{g}$. Then

$$
\operatorname{tr}\left(\mathcal{J} \operatorname{ad}_{X}\right)=0, \text { for any } X \in \mathfrak{g} .
$$

\section{Proof: Let}

$$
\omega_{1}, \ldots, \omega_{n} \in \Lambda^{1,0} \mathfrak{g}
$$

satisfy $(2.1)$, and consider $\bar{\omega}_{1}, \ldots, \bar{\omega}_{n} \in \Lambda^{0,1} \mathfrak{g}$. If $X_{1}, \ldots, X_{n}, \bar{X}_{1}, \ldots, \bar{X}_{n}$ is the basis of $\mathfrak{g}_{\mathbb{C}}^{*}$ dual to $\omega_{1}, \ldots, \omega_{n}, \bar{\omega}_{1}, \ldots, \bar{\omega}_{n}$, then the matrix of $\operatorname{ad}_{X_{k}}$ relative to this basis takes the form:

$$
\left(\begin{array}{cc}
A_{k} & * \\
0 & B_{k}
\end{array}\right)
$$

where $\operatorname{tr}\left(A_{k}\right)=0$ and $B_{k}$ is strictly lower triangular. In fact, let $B_{k}=\left(b_{i l}^{k}\right)$. Using (2.1) one obtains

$$
d \bar{\omega}_{i}=\sum_{j<i} \bar{\eta}_{j}^{i} \wedge \bar{\omega}_{j},
$$


then

$$
\begin{aligned}
b_{i l}^{k} & =\bar{\omega}_{i}\left(\left[X_{k}, \bar{X}_{l}\right]\right)=-2 d \bar{\omega}_{i}\left(X_{k}, \bar{X}_{l}\right) \\
& =-2 \sum_{j<i} \frac{1}{2}\left(\bar{\eta}_{j}^{i}\left(X_{k}\right) \bar{\omega}_{j}\left(\bar{X}_{l}\right)-\bar{\eta}_{j}^{i}\left(\bar{X}_{l}\right) \bar{\omega}_{j}\left(X_{k}\right)\right) \\
& =-\sum_{j<i} \bar{\eta}_{j}^{i}\left(X_{k}\right) \bar{\omega}_{j}\left(\bar{X}_{l}\right),
\end{aligned}
$$

since $\bar{\omega}_{j}\left(X_{k}\right)=0$ for any $j, k$. Observe that when $i \leqslant l, \bar{\omega}_{j}\left(\bar{X}_{l}\right)=0$ for all $j<i$, therefore $b_{i l}^{k}=0$ for $i \leqslant l$, and it turns out that $B_{k}$ is strictly lower triangular, as

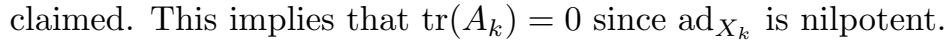

On the other hand, the matrix of $\mathcal{J}$ relative to $X_{1}, \ldots, X_{n}, \bar{X}_{1}, \ldots, \bar{X}_{n}$ is given by:

$$
\left(\begin{array}{cc}
i \mathrm{Id} & 0 \\
0 & -i \mathrm{Id}
\end{array}\right)
$$

therefore, the matrix of $\mathcal{J} \operatorname{ad}_{X_{k}}$ takes the following form:

$$
\left(\begin{array}{cc}
i A_{k} & * \\
0 & -i B_{k}
\end{array}\right)
$$

and, in particular, it has zero trace. A similar argument, using that the matrix of $\operatorname{ad}_{\bar{X}_{k}}$ is given by:

$$
\left(\begin{array}{cc}
C_{k} & 0 \\
* & D_{k}
\end{array}\right)
$$

with $C_{k}$ strictly lower triangular and $\operatorname{tr}\left(D_{k}\right)=0$, gives that $\operatorname{tr}\left(\mathcal{J} \operatorname{ad}_{\bar{X}_{k}}\right)=0$. Therefore, $\operatorname{tr}\left(\mathcal{J} \operatorname{ad}_{X}\right)=0$ for any $X \in \mathfrak{g}_{\mathbb{C}}$ and the lemma follows.

Let $(M, \mathcal{J})$ be a complex manifold, $g$ a Hermitian metric, $\omega=g(\cdot, \mathcal{J} \cdot)$ the Kähler form and $\theta=d^{*} \omega \circ \mathcal{J}$ the Lee form of the Hermitian manifold $(M, \mathcal{J}, g)$, where $d^{*}$ is the adjoint of $d$.

Definition 2.3: A Hermitian metric $g$ on a complex manifold $(M, \mathcal{J})$ is called balanced if $\theta=0$, where $\theta$ is the associated Lee form.

On any Hermitian manifold $(M, \mathcal{J}, g)$ there exists a unique connection $\nabla^{B}$ satisfying $\nabla^{B} g=0, \nabla^{B} J=0$ and whose torsion tensor $c$ (considered as a (3,0)-tensor) $c(X, Y, Z)=g(X, T(Y, Z))$ is totally skew-symmetric. Physicists call this connection a $K T$-connection; among mathematicians this connection is known as the Bismut connection [Bi]. The Lee form can be expressed (locally) in terms of the torsion tensor $c$ as follows (see $[\mathrm{IP}]$ ):

$$
\theta(X)=-\frac{1}{2} \sum_{i=1}^{2 n} c\left(\mathcal{J} X, E_{i}, \mathcal{J} E_{i}\right),
$$

for an orthonormal basis $E_{1}, \ldots, E_{2 n}$ of (local) vector fields.

We restrict next to the case of a left invariant Hermitian structure on a Lie group. The proof of the next lemma follows by using the properties of the Bismut connection together with (2.2). 
Lemma 2.4: Let $G$ be a Lie group with an abelian complex structure $\mathcal{J}$ and $g$ an arbitrary Hermitian left-invariant metric. Then the Bismut connection $\nabla^{B}$ and the Lee form $\theta$ associated to $(G, \mathcal{J}, g)$ are given by

$$
\begin{aligned}
g\left(\nabla_{X}^{B} Y, Z\right) & =-g(X,[Y, Z]), \\
\theta(X) & =\operatorname{tr}\left(\frac{1}{2} J \nabla_{J X}^{B}-\operatorname{ad}_{X}\right),
\end{aligned}
$$

where $X, Y, Z$ are left-invariant vector fields.

2.2. Canonical bundle of a complex nilmanifold. Definition 2.5: Let $N=$ $\Gamma \backslash G$ be a nilmanifold and assume that $G$ is equipped with a left-invariant complex structure. This makes $N$ into a complex manifold. In such a situation we say that $N$ is a complex nilmanifold.

Definition 2.6: A complex structure $\mathcal{I}$ on a nilmanifold $N=\Gamma \backslash G$ is called abelian if it is induced from a left invariant abelian complex structure on $G$.

Theorem 2.7: Let $N=\Gamma \backslash G$ be a complex nilmanifold, $n=\operatorname{dim}_{\mathbb{C}} G$. Then $G$ admits a left-invariant, non-zero, holomorphic section of the canonical bundle $\Lambda^{n, 0}(G)$. In particular, the canonical bundle $K(N)$ of $N$ is trivial, as a holomorphic line bundle.

Proof: Let $\omega_{1}, \ldots, \omega_{n}$ be the left-invariant $(1,0)$-forms and $\eta_{1}^{i}, \ldots, \eta_{i-1}^{i}$ the smooth 1-forms $(2 \leqslant i \leqslant n)$, as in (2.1). If $\eta=\bigwedge_{i=1}^{n} \omega_{i} \in \Lambda^{n, 0}(G)$, we show next that $\eta$ is closed, hence holomorphic. Indeed,

$$
\begin{aligned}
d \eta & =\sum_{i}(-1)^{i+1} \omega_{1} \wedge \cdots \wedge \omega_{i-1} \wedge d \omega_{i} \wedge \omega_{i+1} \wedge \cdots \wedge \omega_{n} \\
& =\sum_{i}(-1)^{i+1} \omega_{1} \wedge \cdots \wedge \omega_{i-1} \wedge\left(\sum_{j<i} \eta_{j}^{i} \wedge \omega_{j}\right) \wedge \omega_{i+1} \wedge \cdots \wedge \omega_{n}=0 .
\end{aligned}
$$

Since $d=\partial+\bar{\partial}$ and $\partial\left(\Lambda^{n, 0}(G)\right) \subset \Lambda^{n+1,0}(G)=0$, it follows that $\bar{\partial} \eta=0$, hence holomorphic.

Finally, the fact that the lattice $\Gamma$ acts on the left implies that left invariant vector fields and 1-forms on $G$ induce global bases of $T N$ and $T^{*} N$ [TO]. Moreover, the canonical projection $\pi: G \rightarrow \Gamma \backslash G$ is holomorphic, hence the last assertion follows.

Another proof of triviality of the canonical bundle of a nilmanifold is found in Theorem 3.1 of $[\mathrm{CG}]$.

On a compact Kähler manifold, topological triviality of the canonical bundle implies that it is trivial holomorphically on some finite, unramified covering of $M$. This follows from Calabi-Yau theorem. Indeed, by Calabi-Yau theorem, $M$ admits a Ricciflat Kähler metric ([Yau]). From Berger's list of irreducible holonomies, de Rham theorem, and Cheeger-Gromoll theorem on fundamental group of Ricci-flat manifolds, we obtain that a finite unramified covering $\widetilde{M}$ of $M$ is a product of compact 
tori, hyperkähler manifolds and simply connected Calabi-Yau manifolds (see [Bes] for a detailed argument). Therefore, $\widetilde{M}$ has trivial canonical bundle.

On a non-Kähler manifold, this is no longer true. However, the above theorem implies that the canonical bundle is holomorphically trivial for every nilmanifold, which is never Kähler unless it is a torus (see [BG]).

For hypercomplex manifolds, $K(M, \mathcal{I})$ is always topologically trivial, which is easy to see by taking a non-degenerate $(2,0)$-form associated with some quaternionic Hermitian structure (Subsection 4.1). The top exterior power of this $(2,0)$-form trivializes $K(M, \mathcal{I})$. However, $K(M, \mathcal{I})$ is quite often non-trivial as a holomorphic line bundle.

It is possible to show that $K(M, \mathcal{I})$ is non-trivial for all hypercomplex manifolds $(M, \mathcal{I}, \mathcal{J}, \mathcal{K})$ such that $(M, \mathcal{I})$ is a principal toric fibration over a base which is a Fano manifold or orbifold (has ample anticanonical bundle). These include the quasiregular locally conformally hyperkähler manifolds (see $[\mathrm{Or}]$ ), which are elliptically fibered over a contact Fano orbifold, and compact Lie groups with the hypercomplex structure constructed by D. Joyce $([\mathrm{J}])$, which are torically fibered over a homogeneous rational manifold ([V3]).

Let $M \stackrel{\pi}{\longrightarrow} B$ be such a fibration. The adjunction formula gives $K(M) \cong \pi^{*} K(B)$, because the canonical bundle of a torus is trivial. However, $\pi^{*} K(B)^{-N}$ has sections, because $K(B)^{-1}$ is ample. Therefore, $K(M)$ can never be trivial.

\section{Hypercomplex nilmanifolds and holonomy}

A manifold $(M, \mathcal{I}, \mathcal{J}, \mathcal{K})$ is called hypercomplex if $\mathcal{I}, \mathcal{J}, \mathcal{K}$ define integrable anticommuting complex structures on $M$ such that $\mathcal{I} \mathcal{J}=\mathcal{K}$. The operators $\mathcal{I}, \mathcal{J}, \mathcal{K}$ define an action of the quaternion algebra $\mathbb{H}$ on the tangent bundle of $M$. As Obata proved ([Ob]), the integrability condition of $\mathcal{I}, \mathcal{J}, \mathcal{K}$ is satisfied if and only if $M$ admits a torsion-free connection $\nabla$ preserving the quaternionic action:

$$
\nabla \mathcal{I}=\nabla \mathcal{J}=\nabla \mathcal{K}=0 .
$$

Such a connection, which is necessarily unique $([\mathrm{Ob}])$, is called the Obata connection on $(M, \mathcal{I}, \mathcal{J}, \mathcal{K})$. Setting $\mathcal{J}_{1}=\mathcal{I}, \mathcal{J}_{2}=\mathcal{J}, \mathcal{J}_{3}=\mathcal{K}$, the Obata connection $\nabla$ is given by (see $[\mathrm{AM}])$ :

$$
\begin{aligned}
\nabla_{X}(Y) & =\frac{1}{12} \sum_{\alpha, \beta, \gamma} \mathcal{J}_{\alpha}\left(\left[\mathcal{J}_{\beta} X, \mathcal{J}_{\gamma} Y\right]+\left[\mathcal{J}_{\beta} Y, \mathcal{J}_{\gamma} X\right]\right) \\
& +\frac{1}{6} \sum_{\alpha=1}^{3} \mathcal{J}_{\alpha}\left(\left[\mathcal{J}_{\alpha} X, Y\right]+\left[\mathcal{J}_{\alpha} Y, X\right]\right)+\frac{1}{2}[X, Y],
\end{aligned}
$$

$X, Y \in \mathfrak{X}(M)$, where $\alpha, \beta, \gamma$ is a cyclic permutation of $1,2,3$.

We consider next hypercomplex nilmanifolds.

Definition 3.1: A hypercomplex structure $\mathcal{I}, \mathcal{J}, \mathcal{K}$ on a Lie group $G$ is called leftinvariant when left translations are holomorphic with respect to the complex structures $\mathcal{I}, \mathcal{J}$ and $\mathcal{K}$. Let $N=\Gamma \backslash G$ be a nilmanifold, with $G$ a Lie group equipped with a left-invariant hypercomplex structure. The quotient $N=\Gamma \backslash G$ inherits a hypercomplex structure. In such situation, we say that $N$ is a hypercomplex nilmanifold. 
Let $\operatorname{Hol}(\nabla)$ be the holonomy group associated with the Obata connection $\nabla$. Since $\nabla$ preserves the quaternionic structure, $\operatorname{Hol}(\nabla) \subset G L(n, \mathbb{H})$. We define the determinant of $h \in G L(n, \mathbb{H})$ in the following way. Let $V \cong \mathbb{H}^{n}$ be the vector space over $\mathbb{H}$, and $V_{I}^{1,0}$ the same space considered as a complex space with the complex structure $I$ induced by $\mathcal{I}$. The Hodge decomposition gives $V \otimes_{\mathbb{R}} \mathbb{C} \cong V_{I}^{1,0} \oplus V_{I}^{0,1}$. The top exterior power $\Lambda_{I}^{2 n, 0}(V):=\Lambda^{2 n}\left(V_{I}^{1,0}\right) \cong \mathbb{C}$ is equipped with a natural real structure:

$$
\eta \longrightarrow J(\bar{\eta})
$$

for $\eta \in \Lambda_{I}^{2 n, 0}(V)$ (the quaternions $I$ and $J$ anticommute, hence $J$ exchanges $\Lambda_{I}^{p, q}(V)$ with $\left.\Lambda_{I}^{q, p}(V)\right)$. Since the real structure on $\Lambda_{I}^{2 n, 0}(V)$ is constructed from the quaternion action, any $h \in G L(V, \mathbb{H})$ preserves this real structure. Let $\operatorname{det}(h)$ denote the action induced by $h$ on $\Lambda_{I}^{2 n, 0}(V) \cong \mathbb{C}$. Then $\operatorname{det}(h) \in \mathbb{R}$, as the above argument implies. This defines a homomorphism

$$
\operatorname{det}: G L(n, \mathbb{H}) \longrightarrow \mathbb{R}^{*}
$$

to the multiplicative group of non-zero real numbers, which is clearly positive since $G L(n, \mathbb{H})$ is connected. Let $S L(n, \mathbb{H}) \subset G L(n, \mathbb{H})$ be the kernel of det, $K(M, \mathcal{I})$ the canonical bundle of $(M, \mathcal{I})$ and $\nabla_{K}$ the connection on $K(M, \mathcal{I})$ induced by the Obata connection $\nabla$. Given $h \in \operatorname{Hol}(\nabla)$, the corresponding transformation in $\operatorname{Hol}\left(\nabla_{K}\right)$ acts on sections of $K(M, \mathcal{I})$ by multiplication by $\operatorname{det}(h)$, hence

$$
\operatorname{Hol}\left(\nabla_{K}\right)=\{\operatorname{det}(h): h \in \operatorname{Hol}(\nabla)\} .
$$

Therefore, $\nabla_{K}$ has trivial holonomy if and only if $\operatorname{Hol}(\nabla) \subset S L(n, \mathbb{H})$. Moreover, the last condition implies that $K(M, \mathcal{I})$ is holomorphically trivial (see [V2], Claim 1.2). We show in Corollary 3.3 that the converse of this statement holds in the case that $M$ is a nilmanifold, thereby giving an affirmative answer to a question raised in [V2] The proof of this corollary makes use of Theorem 2.7 and the next result:

Theorem 3.2: Let $(N, \mathcal{I}, \mathcal{J}, \mathcal{K})$ be a hypercomplex nilmanifold, $\operatorname{dim}_{\mathbb{C}} N=2 n$, and $\eta$ a holomorphic, left-invariant section of the canonical bundle $\Lambda^{2 n, 0}(N, \mathcal{I})$. Then $\nabla \eta=0$, where $\nabla$ is the Obata connection.

Proof: Since the Obata connection is torsion-free, $d \eta=\operatorname{Alt}(\nabla \eta)$, where Alt $=\bigwedge$ : $\Lambda^{2 n}(M) \otimes \Lambda^{1}(M) \longrightarrow \Lambda^{2 n+1}(M)$ denotes the exterior product. Since $\eta$ is holomorphic, $\bar{\partial} \eta=0$. The map Alt restricted to $\Lambda^{2 n, 0}(M) \otimes \Lambda^{0,1}(M)$ is an isomorphism; therefore, $\nabla^{0,1} \eta=0$.

Any left-invariant section of $\Lambda_{I}^{2 n, 0}(N)$ is holomorphic, because such a section is unique, up to a constant multiplier. Therefore, $J(\bar{\eta}) \in \Lambda_{I}^{2 n, 0}(N)$ is holomorphic. This gives

$$
\nabla^{0,1} J(\bar{\eta})=0
$$

Since $\nabla$ commutes with $J$, (3.3) implies $\nabla^{0,1} \bar{\eta}=0$. However, $\nabla^{0,1} \bar{\eta}=\overline{\nabla^{1,0} \eta}$, and this gives $\nabla^{1,0} \eta=0$. We proved that $\nabla^{0,1} \eta+\nabla^{1,0} \eta=\nabla \eta=0$.

Comparing Theorem 3.2 with Theorem 2.7, we obtain the following important corollary: 
Corollary 3.3: Let $(N, \mathcal{I}, \mathcal{J}, \mathcal{K})$ be a hypercomplex nilmanifold. Then $\operatorname{Hol}(\nabla) \subset$ $S L(n, \mathbb{H})$, where $\operatorname{Hol}(\nabla)$ is the holonomy of the Obata connection.

Proof: Theorem 2.7 implies that $\Lambda^{2 n, 0}(N, \mathcal{I})$ has a holomorphic section and by Theorem $3.2, \operatorname{Hol}(\nabla) \subset S L(n, \mathbb{H})$ where $\nabla$ is the Obata connection.

As a consequence of the above result it follows that the Obata connection on any hypercomplex nilmanifold is Ricci flat.

Corollary 3.4: Let $(N, \mathcal{I}, \mathcal{J}, \mathcal{K})$ be a hypercomplex nilmanifold. Then the Ricci tensor of the Obata connection vanishes.

We give two proofs of this corollary; the first one is a consequence of Theorem 5.6 in $[\mathrm{AM}]$ and the second one makes use of Lemma 3.2 in [B2]. In both proofs $\nabla$ denotes the Obata connection of the left-invariant hypercomplex structure on $G$, where $N=\Gamma \backslash G$.

First Proof: It follows from Corollary 3.3 that $\operatorname{Hol}(\nabla) \subset S L(n, \mathbb{H})$. It was proved in $[\mathrm{AM}]$, Theorem $5.6^{1}$, that for a simply connected hypercomplex manifold of dimension $4 k, k>1$, the Obata connection $\nabla$ satisfies $\operatorname{Hol}(\nabla) \subset S L(n, \mathbb{H})$ if and only if the Ricci tensor of $\nabla$ vanishes. Therefore, the Ricci tensor of $\nabla$ vanishes on $G$, hence it vanishes on $N$.

Second Proof: Let $\mathfrak{g}$ be the Lie algebra of $G$ and set $\mathcal{J}_{1}=\mathcal{I}, \mathcal{J}_{2}=\mathcal{J}, \mathcal{J}_{3}=\mathcal{K}$. According to Lemma 3.2 in [B2],

$$
\text { Ric } \equiv 0 \quad \text { if and only if } \operatorname{tr}\left(\nabla_{\left[X_{1}, X_{2}\right]}\right)=0, \forall X_{1}, X_{2} \in \mathfrak{g} .
$$

The first step is to show that:

$$
\operatorname{tr}\left(\nabla_{\left[X_{1}, X_{2}\right]}\right)=\operatorname{tr}\left(\mathcal{J}_{\alpha} \operatorname{ad}_{\mathcal{J}_{\alpha}\left[X_{1}, X_{2}\right]}\right), \quad X_{1}, X_{2} \in \mathfrak{g}, \alpha=1,2,3 .
$$

We compute the trace of $\nabla_{\left[X_{1}, X_{2}\right]}($ recall (3.1)):

$$
\begin{aligned}
& \operatorname{tr}\left(\nabla_{\left[X_{1}, X_{2}\right]}\right)=\frac{1}{6} \operatorname{tr}\left(\sum_{\alpha=1}^{3} \mathcal{J}_{\alpha} \operatorname{ad}_{\mathcal{J}_{\alpha}\left[X_{1}, X_{2}\right]}\right) \\
& +\frac{1}{12} \operatorname{tr}\left(\sum_{\alpha, \beta, \gamma}\left(\mathcal{J}_{\alpha} \operatorname{ad}_{\mathcal{J}_{\beta}\left[X_{1}, X_{2}\right]} \mathcal{J}_{\gamma}-\mathcal{J}_{\alpha} a d_{\mathcal{J}_{\gamma}\left[X_{1}, X-2\right]} \mathcal{J}_{\beta}\right)\right),
\end{aligned}
$$

where $\alpha, \beta, \gamma$ is a cyclic permutation of $1,2,3$.

Since

and

$$
\operatorname{tr}\left(\mathcal{J}_{\alpha} \operatorname{ad}_{\mathcal{J}_{\beta}\left[X_{1}, X_{2}\right]} \mathcal{J}_{\gamma}\right)=\operatorname{tr}\left(\mathcal{J}_{\beta} \operatorname{ad}_{\mathcal{J}_{\beta}\left[X_{1}, X_{2}\right]}\right)
$$

$$
\operatorname{tr}\left(\mathcal{J}_{\alpha} \operatorname{ad}_{\mathcal{J}_{\gamma}\left[X_{1}, X_{2}\right]} \mathcal{J}_{\beta}\right)=-\operatorname{tr}\left(\mathcal{J}_{\gamma} \operatorname{ad}_{\mathcal{J}_{\gamma}\left[X_{1}, X_{2}\right]}\right)
$$

\footnotetext{
${ }^{1}$ Theorem 5.6 in $[\mathrm{AM}]$ holds for $n>1$. For $n=1$ it still holds if we assume that $W_{+}=0$, where $W_{+}$is the self-dual part of the Weyl tensor $W$. This assumption is immediate for hypercomplex manifolds, because the hypercomplex structure gives a parallel trivialization of the bundle $\Lambda^{+}(M)$.
} 
it follows that:

$$
\operatorname{tr}\left(\nabla_{\left[X_{1}, X_{2}\right]}\right)=\frac{1}{3} \operatorname{tr}\left(\sum_{\alpha=1}^{3} \mathcal{J}_{\alpha} \operatorname{ad}_{\mathcal{J}_{\alpha}\left[X_{1}, X_{2}\right]}\right) .
$$

We show next that:

$$
\operatorname{tr}\left(\mathcal{J}_{\alpha} \operatorname{ad}_{\mathcal{J}_{\alpha}\left[X_{1}, X_{2}\right]}\right) \text { is independent of } \alpha=1,2,3 .
$$

Set $X=\left[X_{1}, X_{2}\right]$ and let $Y \in \mathfrak{g}$. Observe that:

$$
\begin{aligned}
\operatorname{tr}\left(\mathcal{J}_{\alpha} \operatorname{add}_{\mathcal{J}_{\alpha} X}\right) & =\operatorname{tr}\left(\operatorname{ad}_{\mathcal{J}_{\alpha} X} \mathcal{J}_{\alpha}\right)=-\operatorname{tr}\left(\mathcal{J}_{\gamma} \operatorname{ad}_{\mathcal{J}_{\alpha} X} \mathcal{J}_{\alpha} \mathcal{J}_{\gamma}\right) \\
& =\operatorname{tr}\left(\mathcal{J}_{\gamma} \operatorname{ad}_{\mathcal{J}_{\alpha} X} \mathcal{J}_{\beta}\right) .
\end{aligned}
$$

The integrability of $\mathcal{J}_{\gamma}$ gives:

$$
\mathcal{J}_{\gamma}\left[\mathcal{J}_{\alpha} X, \mathcal{J}_{\beta} Y\right]=\left[\mathcal{J}_{\beta} X, \mathcal{J}_{\beta} Y\right]-\left[\mathcal{J}_{\alpha} X, \mathcal{J}_{\alpha} Y\right]-\mathcal{J}_{\gamma}\left[\mathcal{J}_{\beta} X, \mathcal{J}_{\alpha} Y\right],
$$

which implies that:

$$
\operatorname{tr}\left(\mathcal{J}_{\gamma} \operatorname{ad}_{\mathcal{J}_{\alpha} X} \mathcal{J}_{\beta}\right)=\operatorname{tr}\left(\operatorname{ad}_{\mathcal{J}_{\beta} X} \mathcal{J}_{\beta}\right)-\operatorname{tr}\left(\operatorname{ad}_{\mathcal{J}_{\alpha} X} \mathcal{J}_{\alpha}\right)-\operatorname{tr}\left(\mathcal{J}_{\gamma} \operatorname{ad}_{\mathcal{J}_{\beta} X} \mathcal{J}_{\alpha}\right) .
$$

Using (3.7) we obtain:

$$
\operatorname{tr}\left(\mathcal{J}_{\alpha} \operatorname{ad}_{\mathcal{J}_{\alpha} X}\right)=\operatorname{tr}\left(\operatorname{ad}_{\mathcal{J}_{\beta} X} \mathcal{J}_{\beta}\right)-\operatorname{tr}\left(\operatorname{ad}_{\mathcal{J}_{\alpha} X} \mathcal{J}_{\alpha}\right)+\operatorname{tr}\left(\mathcal{J}_{\beta} \operatorname{ad}_{\mathcal{J}_{\beta} X}\right),
$$

or equivalently,

$$
2 \operatorname{tr}\left(\mathcal{J}_{\alpha} \operatorname{ad}_{\mathcal{J}_{\alpha} X}\right)=2 \operatorname{tr}\left(\mathcal{J}_{\beta} \operatorname{ad}_{\mathcal{J}_{\beta} X}\right)
$$

and (3.6) follows. This implies (3.4), which together with Lemma 2.2 imply that the corollary holds on $G$, hence on $N=\Gamma \backslash G$.

Remark 3.5: Notice that the converse of Corollary 3.3 is not necessarily true. Indeed, the vanishing of the Ricci curvature is equivalent to the flatness of the canonical bundle $K(N)$ of $N$. However, it might have global monodromy, as it happens in the case of the Hopf surface. Corollary 3.3 implies that (for a nilmanifold), $K(N)$ is trivial, both locally and globally.

\section{Quaternionic Hermitian structures on nilmanifolds}

4.1. HKT structures on abelian nilmanifolds. Let $(M, \mathcal{I}, \mathcal{J}, \mathcal{K})$ be a hypercomplex manifold. A quaternionic Hermitian metric $g$ on $M$ is a Riemannian metric which is Hermitian with respect to $\mathcal{I}, \mathcal{J}$ and $\mathcal{K}$. This is equivalent to $g$ being $S U(2)$-invariant with respect to the $S U(2)$-action generated by the group of $S U(2) \cong S U(\mathbb{H}, 1)$ of unitary quaternions,

$$
S U(\mathbb{H}, 1)=\left\{a+b I+c J+d K \mid a^{2}+b^{2}+c^{2}+d^{2}=1\right\} .
$$

Starting from an arbitrary Riemannian metric and averaging over $S U(2)$, we obtain a quaternionic Hermitian metric. Therefore, such metric always exists.

Definition 4.1: Let $(N, \mathcal{I}, \mathcal{J}, \mathcal{K})$ be a hypercomplex nilmanifold, where $N=\Gamma \backslash G$. When the Lie group $G$ is equipped with a left-invariant quaternionic Hermitian metric we say that the hypercomplex nilmanifold $N$ with the induced metric is a quaternionic Hermitian nilmanifold. 
Let $(M, \mathcal{I}, \mathcal{J}, \mathcal{K})$ be a hypercomplex manifold, $g$ a quaternionic Hermitian metric, and $\Omega$ a 2 -form on $M$ constructed from $g$ as follows:

$$
\Omega:=g(\cdot, \mathcal{J} \cdot)+\sqrt{-1} g(\cdot, \mathcal{K} \cdot)
$$

Then, $\Omega$ is a $(2,0)$-form on $(M, \mathcal{I})$ as an elementary linear-algebraic argument implies ([Bes]).

The hyperkähler condition can be written down as $d \Omega=0$ ([Bes]). The HKT condition is weaker:

Definition 4.2: A quaternionic Hermitian metric is called an HKT-metric if

$$
\partial(\Omega)=0,
$$

where $\partial: \Lambda_{\mathcal{I}}^{2,0}(M) \longrightarrow \Lambda_{\mathcal{I}}^{3,0}(M)$ is the Dolbeault differential on $(M, \mathcal{I})$, and $\Omega$ the $(2,0)$-form on $(M, \mathcal{I})$ constructed from $g$ as in (4.1).

Definition 4.3: Let $(N, \mathcal{I}, \mathcal{J}, \mathcal{K})$ be a hypercomplex nilmanifold, where $N=\Gamma \backslash G$. When the Lie group $G$ is equipped with a left-invariant HKT-metric we say that the hypercomplex nilmanifold $N$ with the induced metric is an HKT nilmanifold.

Remark 4.4: It has been shown in $[\mathrm{FG}]$ that existence of any HKT-metric on $(N, \mathcal{I}, \mathcal{J}, \mathcal{K})$, compatible with a left-invariant hypercomplex structure implies existence of a left-invariant one.

Definition 4.5: A hypercomplex nilmanifold $(N, \mathcal{I}, \mathcal{J}, \mathcal{K})$ with $N=\Gamma \backslash G$ is called abelian when $\mathcal{I}, \mathcal{J}, \mathcal{K}$ are induced by left-invariant abelian complex structures on $G$.

In [DF2], it was shown that for each invariant abelian hypercomplex structure on a Lie group, any left-invariant quaternionic Hermitian metric is HKT. This implies that any abelian hypercomplex nilmanifold is HKT. We show next that as a consequence of Theorem 2.7 the converse of this result holds. The case of 2-step nilmanifolds was proved in [DF2].

Theorem 4.6: Let $(N, \mathcal{I}, \mathcal{J}, \mathcal{K}, g)$ be a nilmanifold admitting an HKT-structure. Then it is abelian.

The proof of the above theorem will follow from a Hard Lefschetz isomorphism on the Dolbeault cohomology of $(N, \mathcal{I})$.

Proposition 4.7: Let $(N, \mathcal{I}, \mathcal{J}, \mathcal{K}, g)$ be an HKT nilmanifold and $\Omega$ the corresponding $(2,0)$-form with respect to $\mathcal{I}$ (see (4.1)). Then,

$$
L_{\Omega}^{n-i}: H_{\partial}^{i, 0}(N, \mathcal{I}) \rightarrow H_{\partial}^{2 n-i, 0}(N, \mathcal{I})
$$

is an isomorphism, where $L_{\Omega}([\gamma])=[\Omega \wedge \gamma]$.

Proof: Let $\omega_{1}, \ldots, \omega_{2 n}$ be a basis of invariant $(1,0)$-forms on $N$ as in the proof of Theorem 2.7. Then $\bar{\eta}=\bar{\omega}_{1} \wedge \cdots \wedge \bar{\omega}_{2 n}$ is an invariant section of the line bundle 
$\Lambda^{0,2 n}(N, \mathcal{I})$. Therefore, $\bar{\Omega}^{n}$ is proportional (with a constant factor) to $\bar{\eta}$. Let $\theta$ be the $(1,0)$-form defined by

$$
\partial \bar{\Omega}^{n}=\theta \wedge \bar{\Omega}^{n} .
$$

Since $\bar{\eta}$ is closed, $\mathrm{d} \bar{\Omega}^{n}=0$, hence $\partial \bar{\Omega}^{n}=0$ and it follows that $\theta=0$. This says that the Dolbeault complex of the square root of the canonical bundle $K(N, \mathcal{I})$ determined by the trivialization induced by $\Omega^{n}$ is identified with the complex $\left(\Lambda^{*, 0}(N, \mathcal{I}), \partial\right)$. The proposition now follows from Theorem 10.2 in [V1].

As a consequence of the above result and Lemma 9 in $[\mathrm{CF}]$ we obtain:

Corollary 4.8: Let $(N, \mathcal{I}, \mathcal{J}, \mathcal{K}, g)$ be an HKT nilmanifold, with $N=\Gamma \backslash G$. Then,

$$
L_{\Omega}^{n-i}: H_{\partial}^{i, 0}\left(\mathfrak{g}_{\mathbb{C}}, I\right) \rightarrow H_{\partial}^{2 n-i, 0}\left(\mathfrak{g}_{\mathbb{C}}, I\right)
$$

is an isomorphism, where $\mathfrak{g}_{\mathbb{C}}$ is the complexification of the Lie algebra of $G$.

Proof of Theorem 4.6: The aim is to show that $\mathfrak{g}^{1,0}$ is abelian. If $\mathfrak{g}^{1,0}$ were not abelian, an analogous argument to that in $[\mathrm{BG}]$ would give that

$$
L_{\Omega}^{n-1}: H_{\partial}^{1,0}(N, \mathcal{I}) \rightarrow H_{\partial}^{2 n-1,0}(N, \mathcal{I})
$$

is not surjective; this contradicts Corollary 4.8. Therefore, $\mathfrak{g}^{1,0}$ must be abelian. Repeating the argument with $\mathcal{J}$ and $\mathcal{K}$ the theorem follows.

The next corollary is a straightforward consequence of Theorem 4.6 (compare with Theorem 3.1 in [DF2]):

Corollary 4.9: The hypercomplex structure of a left-invariant HKT-metric on a nilpotent Lie group admitting a lattice is abelian.

4.2. Quaternionic balanced metrics. Definition 4.10: A quaternionic Hermitian metric $g$ on a hypercomplex manifold is called quaternionic balanced if it is balanced with respect to all complex structures.

Proposition 4.11: Let $(N, \mathcal{I}, \mathcal{J}, \mathcal{K}, g)$ be a quaternionic Hermitian nilmanifold such that the hypercomplex structure is abelian. Then $g$ is quaternionic balanced.

Proof: Let $N=\Gamma \backslash G$; we still denote by $\mathcal{I}, \mathcal{J}, \mathcal{K}, g$ the induced left-invariant quaternionic Hermitian structure on $G$. As shown in [DF2], $g$ is HKT. Therefore, the Bismut connections associated with $\mathcal{I}, \mathcal{J}, \mathcal{K}$ are equal (this is one of the alternative definitions of HKT-structures, see [GP] for details). Denote the Bismut connection of $N$ by $\nabla^{B}$.

Since $G$ is nilpotent, formula (2.3) implies that the Lee form $\theta_{J}$ corresponding to $(J, g)$ is given by

$$
\theta_{J}(X)=\operatorname{tr}\left(\frac{1}{2} J \nabla_{J X}^{B}\right), \quad X \in \mathfrak{g},
$$

where $J$ is the complex structure on $\mathfrak{g}$ induced by $\mathcal{J}$. We show next that $\operatorname{tr}\left(J \nabla_{J X}^{B}\right)=$ 0 . Let $X_{1}, I X_{1}, J X_{1}, K X_{1}, \ldots, X_{n}, I X_{n}, J X_{n}, K X_{n}$ be an orthonormal basis of $\mathfrak{g}$. 
From the definition of the Bismut connection it follows

$$
\nabla^{B} I=\nabla^{B} J=\nabla^{B} K=0 .
$$

Then

$$
\begin{aligned}
\operatorname{tr}\left(J \nabla_{J X}^{B}\right)= & \sum_{j=1}^{n} g\left(J \nabla_{J X}^{B} X_{j}, X_{j}\right)+\sum_{j=1}^{n} g\left(J \nabla_{J X}^{B} I X_{j}, I X_{j}\right) \\
& +\sum_{j=1}^{n} g\left(J \nabla_{J X}^{B} J X_{j}, J X_{j}\right)+\sum_{j=1}^{n} g\left(J \nabla_{J X}^{B} K X_{j}, K X_{j}\right) \\
= & \sum_{j=1}^{n} g\left(J \nabla_{J X}^{B} X_{j}, X_{j}\right)+\sum_{j=1}^{n} g\left(J I \nabla_{J X}^{B} X_{j}, I X_{j}\right) \\
& +\sum_{j=1}^{n} g\left(\nabla_{J X}^{B} J X_{j}, X_{j}\right)+\sum_{j=1}^{n} g\left(J K \nabla_{J X}^{B} X_{j}, K X_{j}\right) \\
= & \sum_{j=1}^{n} g\left(J \nabla_{J X}^{B} X_{j}, X_{j}\right)-\sum_{j=1}^{n} g\left(I J \nabla_{J X}^{B} X_{j}, I X_{j}\right) \\
& +\sum_{j=1}^{n} g\left(\nabla_{J X}^{B} J X_{j}, X_{j}\right)-\sum_{j=1}^{n} g\left(K J \nabla_{J X}^{B} X_{j}, K X_{j}\right) \\
= & \sum_{j=1}^{n} g\left(\nabla_{J X}^{B} J X_{j}, X_{j}\right)-\sum_{j=1}^{n} g\left(\nabla_{J X}^{B} J X_{j}, X_{j}\right) \\
& \sum_{j=1}^{n} g\left(\nabla_{J X}^{B} J X_{j}, X_{j}\right)-\sum_{j=1}^{n} g\left(\nabla_{J X}^{B} J X_{j}, X_{j}\right)=0,
\end{aligned}
$$

therefore, $\theta_{J}(X)=0$ and $g$ is balanced with respect to $J$. The same proof holds for $I$ and $K$.

As a consequence of Theorem 4.6 and Proposition 4.11 we obtain:

Corollary 4.12: Let $(N, \mathcal{I}, \mathcal{J}, \mathcal{K}, g)$ be an HKT nilmanifold. Then $g$ is quaternionic balanced.

4.3. A family of non-abelian hypercomplex nilmanifolds. We end this section by exhibiting a family of hypercomplex nilmanifolds which do not admit HKT metrics. This will follow from Theorem 4.6 since such hypercomplex nilmanifolds are not abelian.

Let $A$ be a finite dimensional associative algebra and $\mathfrak{a f f}(A)$ the Lie algebra $A \oplus A$ with Lie bracket given as follows:

$$
\left[(a, b),\left(a^{\prime}, b^{\prime}\right)\right]=\left(a a^{\prime}-a^{\prime} a, a b^{\prime}-a^{\prime} b\right), \quad a, b, a^{\prime}, b^{\prime} \in A .
$$

These Lie algebras have been considered in [BD2]. We note that $\mathfrak{a f f}(A)$ is a nilpotent Lie algebra if and only if $A$ is nilpotent as an associative algebra.

Let $J$ be the endomorphism of $\mathfrak{a f f}(A)$ defined by

$$
J(a, b)=(b,-a), \quad a, b \in A .
$$


A computation shows that $J$ defines a complex structure on $\mathfrak{a f f}(A)$. Furthermore, if one assumes the algebra $A$ to be a complex associative algebra, this extra assumption allows us to equip $\mathfrak{a f f}(A)$ with a pair of anti-commuting complex structures. Indeed, the endomorphism $K$ on $\mathfrak{a f f}(A)$ defined by $K(a, b)=(-i a, i b)$ for $a, b \in A$ is a complex structure anticommuting with $J$, hence, $J$ and $K$ define a hypercomplex structure by setting $I=J K$. Moreover, the hypercomplex structure is abelian if and only if $A$ is commutative. It then follows that the simply connected Lie groups with Lie algebra $\mathfrak{a} \mathfrak{f f}(A)$, where $A$ is a complex associative non-commutative algebra carry non-abelian hypercomplex structures. In particular, let $A_{k}$ be the algebra of $k \times k$ strictly upper triangular matrices with complex entries and $\operatorname{Aff}\left(A_{k}\right)$ the simply connected Lie group with Lie algebra $\mathfrak{a f f}\left(A_{k}\right)$, which is $(k-1)$-step nilpotent. Since the structure constants with respect to the standard basis of $\mathfrak{a f f}\left(A_{k}\right)$ are integers, there exists a lattice $\Gamma_{k}$ in $\operatorname{Aff}\left(A_{k}\right)$, thus the hypercomplex nilmanifold $N_{k}=\Gamma_{k} \backslash \operatorname{Aff}\left(A_{k}\right)$ does not carry an HKT-metric.

Acknowledgements: Misha Verbitsky thanks Geo Grantcharov and Maxim Kontsevich for interesting discussions of Bogomolov-Tian-Todorov theorem. We are grateful to Maxim Kontsevich for the reference to [G].

\section{References}

[Ab] E. Abbena, An example of an almost Kähler manifold which is not Kählerian, Boll. Un. Mat. Ital. A (6) 3 (1984), no. 3, 383-392.

[AM] D.V. Alekseevsky, S. Marchiafava, Quaternionic structures on a manifold and subordinated structures, Annali di Matematica Pura ed Applicata (IV), Vol. CLXXI (1996), 205-273.

[BK] S. Barannikov, M. Kontsevich, Frobenius Manifolds and Formality of Lie Algebras of Polyvector Fields, alg-geom/9710032, Internat. Math. Res. Notices 1998, no. 4, 201-215.

[B1] M.L. Barberis, Lie groups admitting left invariant hypercomplex structures, 1994, Ph. D. dissertation, National University of Córdoba, Argentina

[B2] M. L. Barberis, Affine connections on homogeneous hypercomplex manifolds, J. Geom. and Physics 32 (1999), 1-13.

[B3] M. L. Barberis, Abelian hypercomplex structures on central extensions of $H$-type Lie algebras, J. Pure Appl. Algebra 158 (2001), 15-23.

[BDM] M.L. Barberis, I. Dotti, R. Miatello, On certain locally homogeneous Clifford manifolds, Ann. Glob. Anal. Geom. 13 (1995), 289-301.

[BD1] M.L. Barberis, I. Dotti, Hypercomplex structures on a class of solvable Lie groups, Quart. J. Math. Oxford Ser. (2) 47 (1996), no. 188, 389-404.

[BD2] M.L. Barberis, I. Dotti, Abelian complex structures on solvable Lie algebras, J. Lie Theory 14(1) (2004), 25-34.

[BG] C. Benson, C.S. Gordon, Kähler and symplectic structures on nilmanifolds, Topology 27(4) (1988) 513-518.

[Bes] A. Besse, Einstein Manifolds, Springer-Verlag, New York (1987).

[Bi] J.M. Bismut, A local index theorem for non-Kähler manifolds, Math. Ann. 284 (1989), 681-699.

[Bo] C.P. Boyer, A note on hyper-Hermitian four-manifolds. Proc. Amer. Math. Soc. 102 (1988), no. 1, 157-164.

[BS] Banos, Bertrand; Swann, Andrew; Potentials for hyper-Kähler metrics with torsion, arXiv:math/0402366, Classical Quantum Gravity 21 (2004), no. 13, 3127-3135.

[CG] Gil R. Cavalcanti, Marco Gualtieri, Generalized complex structures on nilmanifolds, arXiv:math/0404451, J. Symplectic Geom. 2 (2004), no. 3, 393-410. 
[CF] S. Console, A. Fino, Dolbeault cohomology of compact nilmanifolds, Transformation Groups 6 (2) (2001), 111-124.

[CFP] S. Console, A. Fino, Y.S. Poon, Stability of abelian complex structures, Internat. J. Math. 17 (2006), no. 4, 401-416.

[CFG1] L.A. Cordero, M. Fernández, A. Gray, Symplectic manifolds with no Kähler structure, Topology 25 (1986), no. 3, 375-380.

[CFG2] L.A. Cordero, M. Fernńdez, A. Gray, The Frölicher spectral sequence and complex compact nilmanifolds, C. R. Acad. Sci. Paris Sér. I Math. 305 (1987), no. 17, 753-756.

[CFL] L.A. Cordero, M. Fernández, M. de León, Examples of compact complex manifolds with no Kḧler structure, Portugal. Math. 44 (1987), no. 1, 49-62.

[CFGU] L.A. Cordero, M. Fernández, A. Gray, L. Ugarte, Compact nilmanifolds with nilpotent complex structures: Dolbeault cohomology, Trans. Amer. Math. Soc. 352 (12), 5405-5433.

[DF1] I. Dotti, A. Fino, Abelian hypercomplex 8-dimensional nilmanifolds, Ann. Global Anal. Geom. 18 (1) (2000), 47-59.

[DF2] I. Dotti, A. Fino, Hyperkähler torsion structures invariant by nilpotent Lie groups, Class. Quantum Gravity 19 (2002), 551-562, math.DG/0112166.

[DF3] I. Dotti, A. Fino, Hypercomplex nilpotent Lie groups, Contemp. Math. 288 (2001), 310-314.

[Do] A. Douady, Obstruction primaire à la déformation, Sém. H. Cartan 1960/61. 4/01-4/19.

[FG] A. Fino, G. Grantcharov, Properties of manifolds with skew-symmetric torsion and special holonomy, Advances in Math. 189(2) (2004), 429-450, math.DG/0302358.

[G] É. Ghys, Déformations des structures complexes sur les espaces homogènes de $S L(2, C)$, J. Reine Angew. Math. 468 (1995), 113-138.

[GP] G. Grantcharov, Y.S. Poon, Geometry of hyper-Kähler connections with torsion, Comm. Math. Phys. 213 (2000), no. 1, 19-37, math.DG/9908015.

[H] K. Hasegawa, Minimal models of nilmanifolds, Proc. Amer. Math. Soc. 106 (1989), no. 1, 65-71.

[HP] P.S. Howe, G. Papadopoulos, Twistor spaces for hyper-Kähler manifolds with torsion Phys. Lett. B 379 (1996), no. 1-4, 80-86.

[IP] S. Ivanov, G. Papadopoulos, Vanishing theorems and string backgrounds, Class. Quantum Grav. 18 (2001), 1089-1110.

[J] D. Joyce, Compact hypercomplex and quaternionic manifolds, J. Differential Geom. 35 (1992) no. 3, 743-761

[MPPS] C. MacLaughlin, H. Pedersen, Y.S. Poon, S. Salamon, Deformation of 2-step nilmanifolds with abelian complex structures, J. London Math. Soc. (2) 73 (1) (2006), 173-193.

[M] A. I. Mal'čev, On a class of homogeneous spaces, AMS Translation No. 39 (1951).

[MS] S. Merkulov, L. Schwachhöfer, Classification of irreducible holonomies of torsion-free affine connections, math.DG/9907206, Ann. of Math. (2) 150 (1999), no. 1, 77-149, also see Addendum: math.DG/9911266, Ann. of Math. (2) 150 (1999), no. 3, 1177-1179

[Ob] M. Obata, Affine connections on manifolds with almost complex, quaternionic or Hermitian structure, Jap. J. Math., 26 (1955), 43-79.

[Or] L. Ornea, Weyl structures on quaternionic manifolds. A state of the art, math.DG/0105041, also in: Selected Topics in Geomety and Mathematical Physics, vol. 1, 2002, 43-80, E. Barletta ed., Univ. della Basilicata (Potenza).

[PP] H. Pedersen, Y.S. Poon, Inhomogeneous hypercomplex structures on homogeneous manifolds, J. Reine Angew. Math. 516 (1999), 159-181.

[Pe] P. Petravchuk, Lie algebras decomposable as a sum of an abelian and a nilpotent subalgebra, Ukr. Math. J. 40(3) (1988), 385-388.

[Po] Y.S. Poon, Extended deformation of Kodaira surfaces, math/0402440, J. Reine Angew. Math. 590 (2006), 45-65.

[Re] Retakh, V. Massey operations, obstructions to formality in Sullivan's sense and obstructions to Kodaira-Spencer deformations, Uspekhi Mat. Nauk 35 (1980), no. 3(213), 217-220.

[Ro1] S. Rollenske, Some very non-Kähler manifolds: the Frölicher spectral sequence can be arbitrarily non-degenerate, arXiv:0709.0481, 5 pages.

[Ro2] S. Rollenske, The Kuranishi-space of complex parallelisable nilmanifolds, arXiv:0803.2048, 20 pages. 
[SSTV] Ph. Spindel, A. Sevrin, W. Troost, A. Van Proeyen Extended supersymmetric $\sigma$-models on group manifolds, Nucl. Phys. B308 (1988) 662-698.

[S] S.M. Salamon, Complex structures on nilpotent Lie algebras, J. Pure Appl. Algebra 157 (2001), 311-333.

[TO] A. Tralle, J. Oprea, Symplectic manifolds with no Kähler structure. Lecture Notes in Mathematics, 1661. Springer-Verlag, Berlin, 1997. viii+207 pp. ISBN: 3-540-63105-4.

[V1] M. Verbitsky, Hyperkähler manifolds with torsion, supersymmetry and Hodge theory, math.AG/0112215, 47 pages, also in Asian J. of Math., Vol. 6 (4), pp. 679-712 (2002).

[V2] M. Verbitsky, Hypercomplex manifolds with trivial canonical bundle and their holonomy, 15 pages, arXiv:math/0406537, "Moscow Seminar on Mathematical Physics, II", American Mathematical Society Translations, 2, 221 (2007).

[V3] M. Verbitsky, Positive toric fibrations, arXiv:math/0703162, 21 pages.

[Yau] S.T. Yau, On the Ricci curvature of a compact Kähler manifold and the complex MongeAmpère equation I., Comm. on Pure and Appl. Math. 31, 339-411 (1978).

María L. Barberis, Isabel G. Dotti, FamaF - Universidad Nacional de Córdoba, Ciem - Conicet,, Ciudad Universitaria, 5000 Córdoba, Argentina

E-mail address: barberis@mate.uncor.edu, idotti@mate.uncor.edu

Misha Verbitsky, Institute of Theoretical and Experimental Physics, B. CheremushkinSKAYA, 25, Moscow, 117259, Russia

E-mail address: verbit@mccme.ru 